\title{
The Time Has (Finally) Come for the Digital Hospital
}

As anyone with the slightest connection to the healthcare sector knows, hospitals are unique environments. They are the cornerstones of our communities and they provide for the well-being of our citizens.

Physically, they are complicated places, with buildings, people and a vast amount of sophisticated equipment. Publicly, they are under constant scrutiny and pressure to become more patient-centred. Financially, they face a near-constant state of having to do more with less.

Understandably then, the technology requirements of hospitals are unlike those encountered in any other sector.
- simply could not deliver on the promise of complete integration, greater efficiency, at less cost, the Digital Hospital is now finally a reality.

\section{The Digital Hospital Arrives}

Serving a regional population of some 650,000 people, the 1,000-bed St. Olav's Hospital - a health enterprise and university hospital located in the mid-Norway health region near Trondheim, Norway - has become one of the world's first hospitals to implement a Digital Hospital Infrastructure or DHI.

In some respects, it was a matter of technology advancing to the point where specific developments - in this case, the robust Internet Protocol or IP Network and the increased functionality of wireless devices - matured to the point where both widespread applicability and a reasonable cost have now become accessible.

The IP net-work at St. Olav's has con-verted its 11 buildings and grounds into one massive "hotspot" where-in a single central network can be wirelessly accessed by a variety of devices - transportation robots, laptops

For quite some time, the ideal hospital was envisioned to be unified, well-equipped technologically and multi-functional; however, for a variety of reasons - not the least of which is budgetary restraint - hospital IT environments have featured piecemeal implementations, which might satisfy financial management concerns, but do little to increase patient comfort and care or meet increasingly sophisticated departmental needs.

After many false starts, stretching back at least two decades, where the existing technologies - both hardware and software and telephones.

To ensure maximum usage and buy-in, every employee at $\mathrm{St}$. Olav's has an IP phone and access to a PC. The telephone offers direct and efficient communication including patient signal, and, in the near future, staff may view patient information and day-to-day task lists as well as receive notifications.

And when it comes to collaboration, data created by vital hospital equipment and patient monitoring devices, machines like the ECG - which gather and monitor information but often 
can only display it locally - a DHI enables this data to travel seamlessly from one instrument and be made available to user output devices.

For teaching purposes, the network is also enabling realtime learning. Through the DHI, students and aspiring surgeons alike are given remote access to operating theatres via broadcast access points situated in the complex's auditorium. These events are made available live or in a recorded format, and allow students to get a firsthand look at medical procedures in-action.

\section{Acting on the Data}

While comprehensive record keeping is paramount to the healthcare sector, patient records are, if you will, an "after the fact" application, an account of what has happened and only as

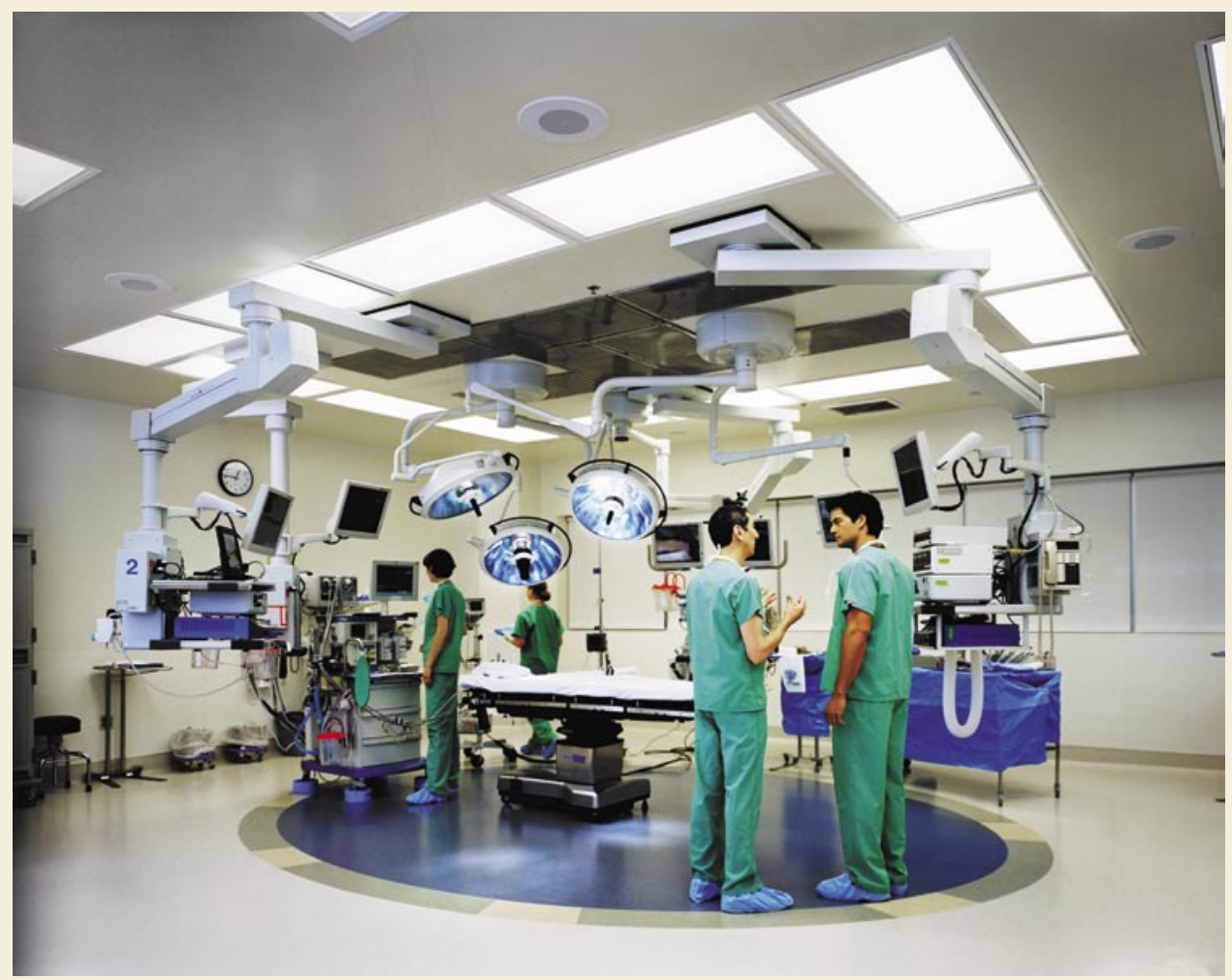

The Digital Hospital Infrastructure enables data to travel seamlessly from one instrument to another and be made available to user output devices, such as broadcast access points allowing medical students remote access to operating theatres. current as the last entry. They cannot actually deliver a service in real-time to patients. Part of the appeal of the DHI is that an IP-based network can act in real-time to govern the dispatch and monitoring of healthcare professionals on an as-required basis.

For example, with the Nurse Call application, when someone triggers a bedside alarm button, a message is sent to a dedicated server that shows the location of the pressed button on one or more Nurse Station displays, while instantly sending a message to the Nurse Call Process, locating the nurse assigned and sending an alarm message to the nurse's mobile device. It's like having a medical professional constantly at a patient's bedside.

There's also an application for hospital orderlies that assigns duties such as moving a patient from one place to another or taking a drink to a patient. Taking into account proximity and workload, this application streamlines the delivery of routine tasks.

For the patient as well, the DHI can deliver impressive features through a Patient Monitor - everything from streaming video to high-speed Internet, to telephone access and food orders. The end result of such convenience and connection is that patients feel less isolated and more entertained or occupied during their stay.

"When it comes to technological infrastructure, St. Olav's
Hospital will probably be one of the world's most advanced for some time to come. Among other things, we are the first hospital in the world to operate all data, TV, radio and voice communication via IP," notes Tore Indrerak, Head of ICT for Helsebygg Midt-Norge, the healthcare authority for the central Norwegian area.

Given this functionality, medical practitioners and support personnel are finding that the time wasted in locating staff not on duty is much reduced. For example, calls can be made to the "anesthetist on duty" without consulting duty rosters; as such, getting service to the end user - the patient - happens in a much more timely fashion.

Over the coming months and years, there will doubtless be many more discrete medical and administrative applications developed. With the open systems architecture of the $\mathrm{DHI}$ and the Internet, they will easily be integrated into the infrastructure.

\section{The Team Delivering DHI}

Hewlett-Packard has assembled and leads a consortium of companies - each expert in its respective field - to provide all the building blocks of an integrated Digital Hospital Infrastructure. 
Based on its successful experience at St. Olav's, HP brought Cisco Systems on board to provide the communications backbone - Cisco's Medical Grade Network, designed specifically for healthcare environments, which demand high availability and rapid response times.

Cardiac Medical AS is a premier designer of medical devices and software. Its IMATIS Inte-gration Platform is an integration engine that compiles, collates and visually presents massive amounts of current information from networks, data sources and equipment anywhere in the hospital. Through rules, modelling and forecasting defined by an individual hospital's own policies and procedures, this information is highly usable and immediately actionable.

\section{Security, Availability Issues}

Security features (i.e., levels of access) are inherent in any IP system that meets the stringent legal and ethical needs for patient record confidentiality. Pre-set parameters to digital records are routinely simpler to enforce than monitoring physical exposure to paper-based files, so patients and administrators can be assured that only those who need to know have access to files.

Clearly, system availability within the 24/7 healthcare environment is crucial; system downtime is simply not an option. St. Olav's has duplicate or redundant fault tolerant systems at every point.

\section{Core Benefits}

In addition to significantly reducing "running" time, a key benefit of the DHI for healthcare professionals is a marked decrease in the laborious, often error-prone manual processes.

Less paperwork means more patient care.

The DHI enables a reduced stress level by eliminating overhead paging and visible/noisy patient signals. With nurses receiving patient calls directly and patients all enjoying IP-based entertainment terminals in their single rooms, the entire environment is more comfortable and less anxious, which can help speed patient recovery and release, while reducing stress and strengthening trust and confidence in the outcomes of their stay.

For hospital administrators, there are a number of concerns that a true DHI addresses. Most important, productivity increases with all the financial and operational benefits such improvement entails. Precious human and dollar resources are put to better use because of improved access to information, less duplication of effort, faster response times and shorter patient stays. Moreover, increased control over physical assets results in better utilization rates and tracking.

Looking forward, a DHI is clearly a long-term solution because, simply, the Ethernet does not age. Being open standards and Internet-based, the DHI can reach back, to incorporate legacy systems and devices, thus protecting investments already made. At the same time, it can reach forward to accommodate future developments that may only be a notion today.

This inherent flexibility means that return on investment is rapid, and that new applications can be brought on-line as appropriate and with the full knowledge they can be integrated into the existing infrastructure without major retrofitting.

Operationally, hospitals with a DHI will experience cleaner workflows and processes by eliminating bottlenecks and achieving quicker responses with better information upon which to make more informed decisions. At the macro-level, administrators have a better overview of workloads and requirements so that they may allocate human resources more effectively.

\section{A DHI is clearly a long-term solution because, simply, the Ethernet does not age. Being open standards and Internet-based, the DHI can reach back, to incorporate legacy systems and devices, thus protecting investments already made. At the same time, it can reach forward to accommodate future developments that may only be a notion today.}

Understandably, over the years, hospitals have been frustrated and consequently suspicious of unfulfilled promises concerning the "next" great thing in healthcare delivery and administration. Equally reasonable is the continual demand for affordable, sustainable healthcare technologies to meet ever-tightening budget considerations.

The answer, we believe, and, best of all now a reality, is the Digital Hospital Infrastructure - a working solution for the future, today.

For more information on HP's healthcare solutions, please visit www.hp.calhealth. 